\title{
Pharmacy Bulk Vial
}

National Cancer Institute

\section{Source}

National Cancer Institute. Pharmacy Bulk Vial. NCI Thesaurus. Code C43212.

A container of a sterile preparation whose contents are intended for use in a pharmacy admixture program and are restricted to the preparation of admixtures for infusion or, through a sterile transfer device, for the filling of empty sterile syringes. 